\begin{tabular}{c|c|c|} 
SELECCIONES MATEMÁTICAS \\
Universidad Nacional de Trujillo \\
ISSN: 2411-1783 (Online) \\
2021; Vol. 8(1): 93-99.
\end{tabular}

\title{
A Demonstration of the Cayley Theorem for Groups
}

\section{Una Demostración del Teorema de Cayley para Grupos}

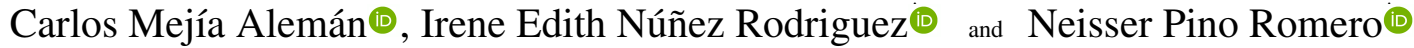 \\ Received, Dec. 25, 2020 \\ Accepted, Apr. 23, 2021

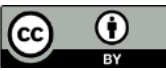

How to cite this article:

Majia CA. et al. A Demonstration of the Cayley Theorem for Groups. Selecciones Matemáticas. 2021;8(1):93-99. http: //dx.doi.org/10.17268/sel.mat.2021.01.09

\begin{abstract}
In this work, we will prove Cayley theorem, using category theory. We will see a different proof than the one seen in a Group Theory course, in this case we will use a famous result in category theory called the Yoneda lemma and then, to relate groups with such lemma we will take advantage of the fact that every group can be viewed as a category. This proof shows that Yoneda lemma is an extensive generalization of Cayley theorem for groups.

Keywords . Categories, functor, natural transformation, Yoneda's Lemma, Cayley's Theorem.

\section{Resumen}

En este trabajo, probaremos el teorema de Cayley para grupos utilizando la teoría de categorías. Veremos una prueba diferente a la que se ve en un curso de Teoría de Grupos, en este caso utilizaremos un famoso resultado en la teoría de categorías que tiene por nombre el Lema de Yoneda, y luego para relacionar grupos con dicho lema nos aprovecharemos de que todo grupo puede ser visto como una categoría. Esta demostración nos hace ver que el lema de Yoneda es una extensa generalización del teorema de Cayley para grupos.
\end{abstract}

Palabras clave. Categoría, funtor, transformación natural, lema de Yoneda, teorema de Cayley.

1. Introducción. En este artículo desarrollaremos la teoría de categorías, utilizadas por primera vez por S. Eilemberg y S. Mac Lane en 1941, ver [2]. Como primera definición veremos que una categoría consiste de una colección de objetos y un conjunto de morfismos que cumplen ciertas condiciones. La palabra colección en este trabajo no necesariamente es un conjunto, pues no existe ningún conjunto que tenga por elementos a todos los conjuntos. Luego veremos como se relacionan objetos y morfismos de una categoría $\mathfrak{C}$ a otra $\mathfrak{D}$; la idea es similar a lo que hace una función $f: A \rightarrow B$, que relaciona elementos de un conjunto A con los de un conjunto B, pero en este caso no son conjuntos sino categorías, a este relacionamiento de objetos y morfismos con ciertas propiedades se le conoce como funtor.

Hay dos tipos de funtores, los covariantes y los contravariantes; nosotros trabajaremos con los del segundo tipo, para más información ver [4].También podemos relacionar funtores contravariantes; es decir, podemos definir $\tau: \mathcal{F} \rightarrow \mathcal{G}$ donde $\mathcal{F}, \mathcal{G}: \mathfrak{C} \rightarrow \mathfrak{D}$ son funtores contravariantes y $\mathfrak{C}, \mathfrak{D}$ categorías. En este caso $\tau$ con ciertas propiedades, es llamada transformación natural. El concepto de transformación natural fue una de las grandes motivaciones para la creación de la teoría de categorías en los trabajos de Saunders Mac Lane y Eilenberg en cohomología.

Para demostrar el teorema de Cayley para grupos, nos falta ver algo más de la teoría de categorías como el Lema de Yoneda que hace de las categorías una teoría con construcciones y teoremas profundos. El lema

\footnotetext{
*Facultad de Ingeniería, Universidad Nacional José María Arguedas. Andahuaylas, Perú. (cme ji a@una jma.edu • pe).

${ }^{\dagger}$ Programa de Estudios Generales, Universidad de Lima. Lima, Perú. (i nunez@ulima . edu . pe).

${ }^{\ddagger}$ Facultad de Ciencias y Filosofía, Universidad Peruana Cayetano Heredia. Lima, Perú. (neisser.pino@upch.pe).
} 
de Yoneda nos dice que existe una biyección entre los conjuntos $\mathcal{F} X$ y $\operatorname{Mor}_{[\mathfrak{C}, \text { Set }]}\left(\mathcal{H}_{X}, \mathcal{F}\right)$ donde $\mathcal{F}: \mathfrak{C} \rightarrow$ Set es un funtor contravariante, $X \in 0 b(\mathfrak{C})$ y $\operatorname{Mor}_{[\mathfrak{C}, \text { Set }]}\left(\mathcal{H}_{X}, \mathcal{F}\right)$ es el conjunto de las transformaciones naturales de la forma $\tau: \mathcal{H}_{X} \rightarrow \mathcal{F}$, para más información sobre el lema de Yoneda ver [7].

Recordemos que el grupo simétrico sobre un conjunto A, denotado por $S_{A}$, es el grupo formado por las aplicaciones biyectivas de A en sí mismo, bajo la operación de composición de funciones, es decir $S_{A}:=\{\Upsilon: A \rightarrow A \mid \Upsilon$ biyección $\}$. Si $A$ es un conjunto finito con $n$ elementos, entonces el grupo $S_{A}$ es llamado grupo de permutaciones de $n$ elementos, que será denotado por $S_{n}$; es sabido que el orden de este grupo es $n$ ! y no es abeliano para $n \geq 3$. El teorema de Cayley para grupos afirma que todo grupo $G$ es isomorfo a un subgrupo del grupo simétrico $S_{G}:=\{\Upsilon: G \rightarrow G \mid \Upsilon$ biyección $\}$, en el caso de que $G$ sea finito de orden $n$, entonces $G$ es isomorfo a un subgrupo de $S_{n}$. Para utilizar el lema de Yoneda en la prueba del teorema de Cayley, construiremos una categoría a partir de grupos y también probaremos algunos lemas.

2. Preliminares. En esta sección veremos algunas definiciones y ejemplos, así como la prueba del lema de Yoneda.

Definición 2.1. Una categoría $\mathfrak{C}$ consta de una colección $O b(\mathfrak{C})$, los elementos de $O b(\mathfrak{C})$ se llaman objetos y se denotan $A, B, C, D, \ldots$ Para cada par $A, B \in O b(\mathfrak{C})$ se tiene un conjunto denotado $\operatorname{Mor}_{\mathfrak{C}}(A, B)$ a cuyos elementos los llamaremos morfismos. Si $f \in \operatorname{Mor}_{\mathfrak{C}}(A, B)$ denotamos $f: A \rightarrow B$. Para cada terna $A, B, C \in O b(\mathfrak{C})$ se tiene una aplicación

$$
\begin{aligned}
\circ: \operatorname{Mor}_{\mathfrak{C}}(A, B) \times \operatorname{Mor}_{\mathfrak{C}}(B, C) & \longrightarrow \operatorname{Mor}_{\mathfrak{C}}(A, C), \\
(f, g) & \mapsto g \circ f,
\end{aligned}
$$

llamada composición de morfismos, que satisface las siguientes condiciones:

1. Para cualesquiera $f \in \operatorname{Mor}_{\mathfrak{C}}(A, B), g \in \operatorname{Mor}_{\mathfrak{C}}(B, C)$ y $h \in \operatorname{Mor}_{\mathfrak{C}}(C, D)$ se tiene que $h \circ(g \circ$ $f)=(h \circ g) \circ f$. Por lo tanto, la composición de morfismos es asociativa.

2. Para cada $A \in O b(\mathfrak{C})$, existe un morfismo $1_{A} \in \operatorname{Mor}_{\mathfrak{C}}(A, A)$ llamado morfismo identidad, tal que para cualesquiera $f \in \operatorname{Mor}_{\mathfrak{C}}(B, A), g \in \operatorname{Mor}_{\mathfrak{C}}(A, B)$ tenemos que $1_{A} \circ f=f, g \circ 1_{A}=g$.

Ejemplo 2.1. La categoría de conjuntos Set cuyos objetos son los conjuntos y cuyos morfismos son las funciones.

Ejemplo 2.2. La categoría de grupos abelianos $\boldsymbol{A b}$ cuyos objetos son los grupos abelianos y cuyos morfismos son los homomorfismos de grupos abelianos.

El siguiente ejemplo es importante para el resultado central.

Ejemplo 2.3. Todo grupo $(G, \cdot)$ puede ser visto como una categoría que denotaremos por $\mathfrak{C}_{G}$. Esta categoría tiene un solo objeto que lo denotaremos por" * y cuyos morfismos serán los elementos del grupo. En efecto: Vamos a definir la aplicación composición como la operación del grupo G, es decir:

$$
\begin{aligned}
\circ: \operatorname{Mor}_{\mathfrak{C}_{G}}(*, *) \times \operatorname{Mor}_{\mathfrak{C}_{G}}(*, *) & \longrightarrow \operatorname{Mor}_{\mathfrak{C}_{G}}(*, *), \\
(f, g) & \mapsto g \circ f=g \cdot f .
\end{aligned}
$$

Por la operación del grupo tenemos que $(h \circ g) \circ f=(h \cdot g) \cdot f=h \cdot(g \cdot f)=h \circ(g \circ f)$, para cualesquiera $f, g, h: * \rightarrow *$ en $\mathfrak{C}_{G}$ y existe $e: * \rightarrow *$ en $\mathfrak{C}_{G}$ tal que e $\circ f=e \cdot f=f$ y $g \circ e=g \cdot e=g$, para cada $f, g: * \rightarrow *$ en $\mathfrak{C}_{G}$, donde e $\in G$ es el elemento neutro. Por lo tanto $\mathfrak{C}_{G}$ es una categoría.

Ejemplo 2.4. La categoría de anillos conmutativos con unidad CRing cuyos objetos son los anillos conmutativos con unidad y cuyos morfismos son los homomorfismos de anillos conmutativos que respetan la unidad.

Ejemplo 2.5. Sea $R$ un anillo con unidad. La categoría de módulos a izquierda $R$-Mod cuyos objetos son módulos a izquierda y cuyos morfismos son los homomorfismos de R-módulos a izquierda. También tenemos la categoría de módulos a derecha Mod-R cuyos objetos son los $R$-módulos a derecha y cuyos morfismos son los homomorfismos de R-módulos a derecha.

Ejemplo 2.6. Si $\mathbb{K}$ es un cuerpo, tenemos la categoría de los $\mathbb{K}$-espacios vectoriales Vect $_{\mathbb{K}}$ cuyos objetos son los $\mathbb{K}$-espacios vectoriales y cuyos morfismos son las transformaciones lineales.

Ejemplo 2.7. La categoría de espacios topológicos Top cuyos objetos son los espacios topológicos y cuyos morfismos son aplicaciones continuas entre espacios topológicos. 
Para ejemplos más sofisticados se puede revisar [6], y para una descripción de categorías sin hacer uso de la teoría de conjuntos ver el famoso libro de MacLane [4].

Definición 2.2. Sean $\mathfrak{C}$ y $\mathfrak{D}$ categorías. Un funtor contravariante que va de $\mathfrak{C}$ a $\mathfrak{D}$ es denotado por $\mathcal{F}: \mathfrak{C} \rightarrow \mathfrak{D}$ y se define como sigue.

i) Para cada objeto $A \in O b(\mathfrak{C})$ asignamos un objeto $\mathcal{F} A \in O b(\mathfrak{D})$.

ii) Para cada morfismo $f: A \rightarrow B$ en $\mathfrak{C}$ asignamos un morfismo $\mathcal{F} f: \mathcal{F} B \rightarrow \mathcal{F} A$ en $\mathfrak{D}$ tal que

1. Para cada $A \in O b(\mathfrak{C})$ se tiene que $\mathcal{F} 1_{A}=1_{\mathcal{F} A}$.

2. Para $A, B, C \in O b(\mathfrak{C})$ y para $f: A \rightarrow B, g: B \rightarrow C$ morfismos en $\mathfrak{C}$ se tiene que $\mathcal{F}(g \circ f)=$ $\mathcal{F} f \circ \mathcal{F} g$.

Ejemplo 2.8. Para cada $X \in O b(\mathfrak{C})$ definimos $\mathcal{H}_{X}: \mathfrak{C} \rightarrow$ Set de la siguiente manera: Para $U \in$ $O b(\mathfrak{C})$ tenemos que $\mathcal{H}_{X} U:=\operatorname{Mor}_{\mathfrak{C}}(U, X)$ y para un morfismo $f: U \rightarrow V$ en $\mathfrak{C}$ tenemos un morfismo en Set

$$
\begin{aligned}
\mathcal{H}_{X} f: \mathcal{H}_{X} V:=\operatorname{Mor}_{\mathfrak{C}}(V, X) & \longrightarrow \mathcal{H}_{X} U:=\operatorname{Mor}(U, X), \\
g & \mapsto \mathcal{H}_{X} f(g):=g \circ f .
\end{aligned}
$$

Así definido $\mathcal{H}_{X}$ es un funtor contravariante.

Ejemplo 2.9. Definimos $\mathcal{F}:$ Set $\rightarrow$ Set de la siguiente manera: Para cada $A \in O b($ Set $)$ definimos $\mathcal{F} A:=\mathcal{P}(A)$ donde $\mathcal{P}(A)$ es el conjunto potencia de A y para cada morfismo $f: A \rightarrow B$ en Set definimos el morfismo

$$
\begin{aligned}
\mathcal{F} f: \mathcal{F} B & \longrightarrow \mathcal{F} A, \\
B_{1} & \mapsto f^{-1}\left(B_{1}\right) .
\end{aligned}
$$

en Set. Así definido $\mathcal{F}$ es un funtor contravariante.

Definición 2.3. Sean $\mathfrak{C}$ y $\mathfrak{D}$ dos categorías y sean $\mathcal{F}, \mathcal{G}: \mathfrak{C} \rightarrow \mathfrak{D}$ dos funtores contravariantes. Una transformación natural $\tau: \mathcal{F} \rightarrow \mathcal{G}$ es dada por una colección de morfismos $\tau_{A}: \mathcal{F} A \rightarrow \mathcal{G} A$, indexada por los objetos $A \in O b(\mathfrak{C})$ tal que:

1. Para cada objeto $A \in O b(\mathfrak{C})$ existe un morfismo $\tau_{A}: \mathcal{F} A \rightarrow \mathcal{G} A$ en $\mathfrak{D}$.

2. Para cada morfismo $f: A \rightarrow B$ en $\mathfrak{C}$ el siguiente diagrama conmuta en $\mathfrak{D}$.

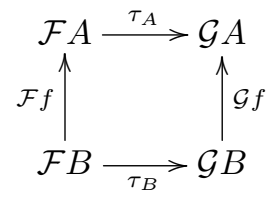

Ejemplo 2.10. Sean $\mathfrak{C}$ y $\mathfrak{D}$ dos categorías y $\mathcal{F}: \mathfrak{C} \rightarrow \mathfrak{D}$ un funtor contravariante. Definimos $\tau: \mathcal{F} \rightarrow$ $\mathcal{F}$ tal que asigna a cada $A \in O b(\mathfrak{C})$ el morfismo identidad $\tau_{A}=1_{\mathcal{F} A}: \mathcal{F} A \rightarrow \mathcal{F} A$ en $\mathfrak{D}$. Así definido $\tau$ es una transformación natural.

Ejemplo 2.11. Sean $\mathfrak{C}$ y $\mathfrak{D}$ dos categorías y $\mathcal{F}, \mathcal{G}, \mathcal{J}: \mathfrak{C} \rightarrow \mathfrak{D}$ tres funtores contravariantes. Si $\tau: \mathcal{F} \rightarrow \mathcal{G}$ y $\eta: \mathcal{G} \rightarrow \mathcal{J}$ son transformaciones naturales entonces $\eta \circ \tau: \mathcal{F} \rightarrow \mathcal{J}$ es una transformación natural si tiene la siguiente propiedad: Para cada $A \in O b(\mathfrak{C})$ tenemos que $(\eta \circ \tau)_{A}=\eta_{A} \circ \tau_{A}$.

En efecto: Vemos que para cada $A \in O b(\mathfrak{C})$ podemos asociar un morfismo $(\eta \circ \tau)_{A}=\eta_{A} \circ \tau_{A}$ en $\mathfrak{D}$. Ahora veamos que para cada morfismo $f: A \rightarrow B$ en $\mathfrak{C}$ el siguiente diagrama conmuta en $\mathcal{D}$.

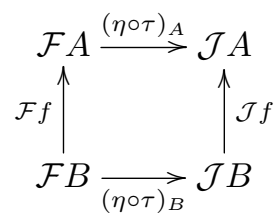

esto es, $\mathcal{J} f \circ(\eta \circ \tau)_{B}=(\eta \circ \tau)_{A} \circ \mathcal{F} f$. Por hipótesis los siguientes digramas conmutan en $\mathcal{D}$ 

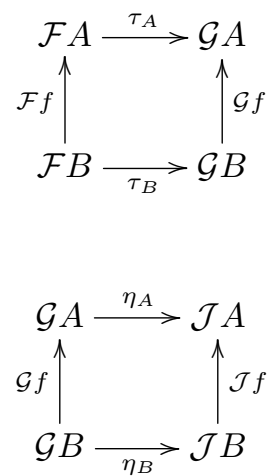

esto quiere decir que $\mathcal{G} f \circ \tau_{B}=\tau_{A} \circ \mathcal{F} f, \mathcal{J} f \circ \eta_{B}=\eta_{A} \circ \mathcal{G} f$. Partimos de $\mathcal{J} f \circ(\eta \circ \tau)_{B}=\mathcal{J} f \circ\left(\eta_{B} \circ \tau_{B}\right)=$ $\left(\mathcal{J} f \circ \eta_{B}\right) \circ \tau_{B}=\left(\eta_{A} \circ \mathcal{G} f\right) \circ \tau_{B}=\eta_{A} \circ\left(\mathcal{G} f \circ \tau_{B}\right)=\eta_{A} \circ\left(\tau_{A} \circ \mathcal{F} f\right)=\left(\eta_{A} \circ \tau_{A}\right) \circ \mathcal{F} f=(\eta \circ \tau)_{A} \circ \mathcal{F} f$. Por lo tanto $\eta \circ \tau: \mathcal{F} \rightarrow \mathcal{J}$ es una transformación natural.

Definición 2.4. Sean $\mathfrak{C}$ y $\mathfrak{D}$ dos categorías y sean $\mathcal{F}, \mathcal{G}: \mathfrak{C} \rightarrow \mathfrak{D}$ dos funtores contravariantes. Las transformaciones naturales $\mu, \tau: \mathcal{F} \rightarrow \mathcal{G}$ son iguales si para cada $A \in O b(\mathfrak{C})$ se tiene que $\mu_{A}=\tau_{A}$.

Ahora vamos a considerar la categoría de los funtores contravariantes que denotaremos por $[\mathfrak{C}, \mathfrak{D}]$ donde los objetos son funtores contravariantes de la forma $\mathcal{F}: \mathfrak{C} \rightarrow \mathfrak{D}$ y los morfismos son transformaciones naturales de la forma $\tau: \mathcal{F} \rightarrow \mathcal{G}$ donde $\mathcal{F}, \mathcal{G}: \mathfrak{C} \rightarrow \mathfrak{D}$. Veamos que en efecto [ $\mathfrak{C}, \mathfrak{D}]$ es una categoría: Vamos a definir la aplicación composición como sigue:

$$
\begin{aligned}
\circ: \operatorname{Mor}_{\mathfrak{C}}(\mathcal{F}, \mathcal{G}) \times \operatorname{Mor}_{\mathfrak{C}}(\mathcal{G}, \mathcal{J}) & \longrightarrow \operatorname{Mor}_{\mathfrak{C}}(\mathcal{F}, \mathcal{J}) \\
(\tau, \eta) & \mapsto \eta \circ \tau .
\end{aligned}
$$

tal que para cada $A \in O b(\mathfrak{C})$ se tiene que $(\eta \circ \tau)_{A}=\eta_{A} \circ \tau_{A}$. Esta aplicación está bien definida por el ejemplo 10. Vemos que $(\tau \circ \eta) \circ \mu=\tau \circ(\eta \circ \mu)$, pues $((\tau \circ \eta) \circ \mu)_{A}=(\tau \circ(\eta \circ \mu))_{A}$, para cada $A \in O b(\mathfrak{C})$ y para cualesquiera $\tau: \mathcal{F} \rightarrow \mathcal{G}, \eta: \mathcal{G} \rightarrow \mathcal{J}$ y $\mu: \mathcal{J} \rightarrow \mathcal{E}$.

Esto quiere decir que la aplicación composición es asociativa. Por otro lado tenemos que para cualquier objeto $\mathcal{F}: \mathfrak{C} \rightarrow \mathfrak{D}$ de la categoría $[\mathfrak{C}, \mathfrak{D}]$, existe un morfismo $1_{\mathcal{F}} \in \operatorname{Mor}_{[\mathfrak{C}, \mathfrak{D}]}(\mathcal{F}, \mathcal{F})$ tal que para cualesquiera $\tau \in \operatorname{Mor}_{[\mathfrak{C}, \mathfrak{D}]}(\mathcal{G}, \mathcal{F})$ y $\eta \in \operatorname{Mor}_{[\mathfrak{C}, \mathfrak{D}]}(\mathcal{F}, \mathcal{G})$ tenemos que $1_{\mathcal{F}} \circ \tau=\tau$ y $\eta \circ 1_{\mathcal{F}}=\eta$, pues $\left(1_{\mathcal{F}} \circ \tau\right)_{A}=\tau_{A} \mathrm{y}\left(\eta \circ 1_{\mathcal{F}}\right)_{A}=\eta_{A}$, para cada $A \in O b(\mathfrak{C})$. Por lo tanto $[\mathfrak{C}, \mathfrak{D}]$ es una categoría.

Proposición 2.1. (Lema de Yoneda) Sea $\mathcal{F}: \mathfrak{C} \rightarrow$ Set un funtor contravariante. Para cualquier $X \in O b(\mathfrak{C})$ se cumple que existe una aplicación biyectiva

$$
T: \mathcal{F} X \quad \longrightarrow \quad \operatorname{Mor}_{[\mathfrak{C}, \text { Set }]}\left(\mathcal{H}_{X}, \mathcal{F}\right)
$$

Demostración: Definimos $T$ de la siguiente manera:

$$
\begin{aligned}
& T: \mathcal{F} X \quad \longrightarrow \quad \operatorname{Mor}_{[\mathfrak{C}, \mathbf{S e t}]}\left(\mathcal{H}_{X}, \mathcal{F}\right), \\
& a \longmapsto \quad T(a):=\tau^{a}
\end{aligned}
$$

tal que para cada $U \in O b(\mathfrak{C})$ se tiene que:

$$
\begin{aligned}
\tau_{U}^{a}: \mathcal{H}_{X} U & \longrightarrow \mathcal{F} U, \\
f & \longmapsto \tau_{U}^{a}(f):=\mathcal{F} f(a) .
\end{aligned}
$$

Vemos que $\tau_{U}^{a}$ está bien definida ya que $f: U \rightarrow X \in \mathcal{H}_{X} U:=\operatorname{Mor}_{\mathfrak{C}}(U, X)$ luego $\mathcal{F} f: \mathcal{F} X \rightarrow \mathcal{F} U$ es un morfismo en Set ya que $\mathcal{F}: \mathfrak{C} \rightarrow$ Set es un funtor contravariante y como $a \in \mathcal{F} X$ entonces $\mathcal{F} f(a) \in \mathcal{F} U$. Ahora veamos que $\tau^{a}: \mathcal{H}_{X} \rightarrow \mathcal{F}$ es una transformación natural. En efecto: dado un morfismo $\alpha: U \rightarrow V$ en $\mathfrak{C}$ vemos que el siguiente diagrama 


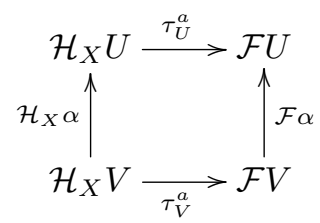

es conmutativo pues $\left(\tau_{u}^{a} \circ \mathcal{H}_{X} \alpha\right)(r)=\tau_{U}^{a}\left(\mathcal{H}_{X} \alpha(r)\right)=\tau_{U}^{a}(r \circ \alpha)=\mathcal{F} r \circ \alpha(a)$ y $\left(\mathcal{F} \alpha \circ \tau_{V}^{a}\right)(r)=$ $\mathcal{F} \alpha\left(\tau_{V}^{a}(r)\right)=\mathcal{F} \alpha(\mathcal{F} r(a))=(\mathcal{F} \alpha \circ \mathcal{F} r)(a)=\mathcal{F} r \circ \alpha(a)$ luego $\tau^{a}$ es una transformación natural. Por lo tanto $T$ está bien definida.

Afirmación 1: $T$ es inyectiva. En efecto: sean $a, b \in \mathcal{F} X$ tales que $\tau^{a}=\tau^{b}$. Tomemos $U=X$ luego

$$
\begin{aligned}
\tau_{X}^{a}: \mathcal{H}_{X} X & \longrightarrow \mathcal{F} X, \\
1_{X} & \longmapsto a,
\end{aligned}
$$

pues $\tau_{X}^{a}\left(1_{X}\right)=\mathcal{F} 1_{X}(a)=1_{\mathcal{F} X}(a)=a$ y dado que $\tau^{a}=\tau^{b}$ entonces $\tau_{X}^{a}=\tau_{X}^{b}$ luego $\tau_{X}^{a}\left(1_{X}\right)=$ $\tau_{X}^{b}\left(1_{X}\right)$ entonces $a=b$. Por lo tanto $T$ es inyectiva.

Afirmación 2: $T$ es sobreyectiva. En efecto: por probar que para cada $\tau \in \operatorname{Mor}_{[\mathfrak{C}, \text { Set }]}\left(\mathcal{H}_{X}, \mathcal{F}\right)$ existe un $a \in \mathcal{F} X$ tal que $\tau_{U}^{a}=\tau_{U}$ para cada $U \in O b(\mathfrak{C})$. Dada $\tau \in M o r_{[\mathfrak{C}, \text { Set }]}\left(\mathcal{H}_{X}, \mathcal{F}\right)$ y un objeto $U \in O b(\mathfrak{C})$.

Consideremos el morfismo $\alpha: U \rightarrow X$ en $\mathfrak{C}$ entonces el siguiente diagrama conmuta

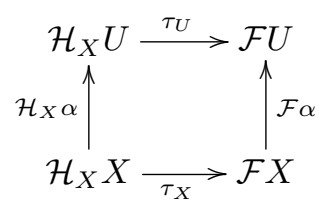

pues $\tau: \mathcal{H}_{X} \rightarrow \mathcal{F}$ es una transformación natural. Tenemos que $\left(\tau_{U} \circ \mathcal{H}_{X} \alpha\right)\left(1_{X}\right)=\tau_{U}\left(\mathcal{H}_{X} \alpha\left(1_{X}\right)\right)=$ $\tau_{U}\left(1_{X} \circ \alpha\right)=\tau_{U}(\alpha)$ pues $1_{X} \in \mathcal{H}_{X} X$ y por otro lado como el diagrama conmuta entonces tenemos la siguiente igualdad $\left(\tau_{U} \circ \mathcal{H}_{X} \alpha\right)=\left(\mathcal{F} \alpha \circ \tau_{X}\right)$ luego $\left(\tau_{U} \circ \mathcal{H}_{X} \alpha\right)\left(1_{X}\right)=\left(\mathcal{F} \alpha \circ \tau_{X}\right)\left(1_{X}\right)=\mathcal{F} \alpha\left(\tau_{X}\left(1_{X}\right)\right)$ y llamemos a $\tau_{X}\left(1_{X}\right):=a \in \mathcal{F} X$ entonces $\tau_{U}^{a}(\alpha):=\mathcal{F} \alpha(a)=\left(\tau_{U} \circ \mathcal{H}_{X} \alpha\right)\left(1_{X}\right)$ luego tenemos que $\tau_{U}(\alpha)=\tau_{U}^{a}(\alpha)$. Por lo tanto $T$ es sobreyectiva.

Para más información sobre el lema de Yoneda se puede revisar [1], [3] y [7].

3. Resultado central. Después de haber presentado las definiciones en la sección 2, procederemos a demostrar el teorema de Cayley para grupos, antes veremos algunos resultados.

Lema 3.1. Sea $\mathfrak{C}$ una categoría, entonces $\operatorname{Mor}_{\mathfrak{C}}(A, A)$ es un monoide con la composición de morfismos.

Demostración: Si $f, g \in \operatorname{Mor}_{\mathfrak{C}}(A, A)$ entonces se tiene que $g \circ f \in \operatorname{Mor}_{\mathfrak{C}}(A, A)$, también se tiene la asociatividad $(h \circ g) \circ f=h \circ(g \circ f)$, ya que el morfismo identidad $1_{A}: A \rightarrow A$ es único tal que $1_{A} \circ f=f \circ 1_{A}=f$ para cada morfismo $f: A \rightarrow A \in \operatorname{Mor}_{\mathfrak{C}}(A, A)$ entonces concluimos que $\operatorname{Mor}_{\mathfrak{C}}(A, A)$ es un monoide. En particular, si $\mathfrak{C}$ es una categoría entonces $\operatorname{Mor}_{[\mathfrak{C}, \operatorname{Set}]}(\mathcal{F}, \mathcal{F})$ es un monoide con la composición de transformaciones naturales.

Lema 3.2. Existe una biyección entre $\mathcal{H}_{*} *$ y $\operatorname{Mor}_{\left[\mathfrak{c}_{G}, S e t\right]}\left(\mathcal{H}_{*}, \mathcal{H}_{*}\right)$.

Demostración: Por el lema de Yoneda tenemos que existe una biyección

$$
\begin{aligned}
& F: \mathcal{H}_{*} * \quad \longrightarrow \quad \operatorname{Mor}_{\left[\mathfrak{C}_{G}, \text { Set }\right]}\left(\mathcal{H}_{*}, \mathcal{H}_{*}\right), \\
& f \longmapsto \quad F(f):=\tau^{f},
\end{aligned}
$$

tal que para cada $* \in \mathfrak{C}_{G}$ se tiene que: 


$$
\begin{array}{rlc}
\tau_{*}^{f}: \mathcal{H}_{*} * & \longrightarrow & \mathcal{H}_{*} * \\
g & \longmapsto & \tau_{*}^{f}(g):=\mathcal{H}_{*} g(f)=f \cdot g .
\end{array}
$$

Lema 3.3. Para cada $f, g \in \mathcal{H}_{*} *$ se tiene que $F(f \circ g)=F(f) \circ F(g)$, donde $F$ es la biyección del lema anterior.

Demostración: La igualdad $F(f \circ g)=F(f) \circ F(g)$ es lo mismo que $\tau^{f \circ g}=\tau^{f} \circ \tau^{g}$ y esto es equivalente a $\tau_{*}^{f \circ g}=\left(\tau^{f} \circ \tau^{g}\right)_{*}:=\tau_{*}^{f} \circ \tau_{*}^{g}$ para cada $* \in C_{G}$, luego probar la igualdad $F(f \circ g)=$ $F(f) \circ F(g)$ es lo mismo que probar $\tau_{*}^{f \circ g}(h)=\left(\tau_{*}^{f} \circ \tau_{*}^{g}\right)(h)$, para cada $h \in \mathcal{H}_{*} *$ y esto último justamente se da pues $\tau_{*}^{f \circ g}(h)=\tau_{*}^{g \cdot f}(h)=h \cdot(g \cdot f)=(h \cdot g) \cdot f=\tau_{*}^{f}(h \cdot g)=\tau_{*}^{f}\left(\tau_{*}^{g}(h)\right)=\left(\tau_{*}^{f} \circ \tau_{*}^{g}\right)(h)$, para cada $h \in \mathcal{H}_{*} *$.

Después de haber enunciado los lemas 3.1, 3.2, y 3.3, demostraremos el Teorema de Cayley para Grupos siendo el resultado central del presente trabajo.

Teorema 3.1. (Teorema de Cayley para Grupos). Todo grupo es isomorfo a un subgrupo de algún grupo simétrico.

Demostración: Sea $(G, \cdot)$ un grupo, para cada $g \in G$ definimos el siguiente automorfismo

$$
\begin{aligned}
& \psi_{g}: G \quad \longrightarrow \quad G, \\
& f \longmapsto \psi_{g}(f):=g \cdot f .
\end{aligned}
$$

Es fácil ver que $\operatorname{Mor}_{\left[\mathfrak{C}_{G}, \text { Set }\right]}\left(\mathcal{H}_{*}, \mathcal{H}_{*}\right) \subset\left\{\psi_{g} \mid g \in G\right\} \subset\{\Upsilon: G \rightarrow G \mid \Upsilon$ biyección $\}$. Por los lemas 3.1, 3.2 y 3.3, tenemos que $F: \mathcal{H}_{*} * \rightarrow \operatorname{Mor}_{\left[\mathfrak{C}_{G}, \text { Set }\right]}\left(\mathcal{H}_{*}, \mathcal{H}_{*}\right)$ es un isomorfismo de monoides y dado que $\mathcal{H}_{*} *=G$, donde $G$ es un grupo entonces $\operatorname{Mor}_{\left[\mathfrak{C}_{G}, \text { Set }\right]}\left(\mathcal{H}_{*}, \mathcal{H}_{*}\right)$ es un grupo, luego obtenemos que $\operatorname{Mor}_{\left[\mathfrak{C}_{G}, \operatorname{Set}\right]}\left(\mathcal{H}_{*}, \mathcal{H}_{*}\right)$ es un subgrupo de $\{\Upsilon: G \rightarrow G \mid \Upsilon$ biyección $\}$.

Por lo tanto el grupo $G$ es isomorfo a un subgrupo del grupo simétrico $S_{G}=\{\Upsilon: G \rightarrow G \mid \Upsilon$ biyección $\}$ y esto es justamente el teorema de Cayley para grupos.

En [5], se da una idea de la prueba del teorema de Cayley para grupos, utilizando un corolario del lema de Yoneda, el funtor covariante $\mathcal{H}^{*}: \mathfrak{C}_{G} \rightarrow$ Set, y la categoría de los funtores covariantes $[\mathfrak{C}, \mathfrak{D}]_{\text {cov }}$, donde los objetos son funtores covariantes de la forma $\mathcal{F}: \mathfrak{C} \rightarrow \mathfrak{D}$ y los morfismos son transformaciones naturales de la forma $\tau: \mathcal{F} \rightarrow \mathcal{G}$ donde $\mathcal{F}, \mathcal{G}: \mathfrak{C} \rightarrow \mathfrak{D}$.

4. Conclusiones. Las categorías con las que hemos trabajado son llamadas categorías localmente pequeñas, es decir aquellas categorías donde $\operatorname{Mor}_{\mathfrak{C}}(A, B)$ es un conjunto. Existen categorías donde la colección de objetos es un conjunto, estas categorías son llamadas pequeñas, como por ejemplo la categoría del ejemplo 3, también existen categorías donde la colección de objetos y la colección de morfismos no son conjuntos, como por ejemplo la categoría de las categorías denotada por Cat, donde los objetos son categorías pequeñas y los morfismos son funtores entre categorías pequeñas, ver [6] y [8].

Gracias a que las transformaciones naturales se pueden componer de forma natural, conseguimos que $[\mathfrak{C}, \mathfrak{D}]$ sea una categoría, luego para enunciar el lema de Yoneda consideramos la categoría [C $\mathfrak{C}$, Set]. Para llegar a probar el teorema de Cayley para grupos, trabajamos con la categoría $\mathfrak{C}_{G}$ y por el lema de Yoneda y los lemas 3.1, 3.2 y 3.3 obtuvimos un isomorfismo de monoides entre $\mathcal{H}_{*} *$ y $\operatorname{Mor}_{\left[\mathfrak{C}_{G}, \operatorname{Set}\right]}\left(\mathcal{H}_{*}, \mathcal{H}_{*}\right)$.

Este trabajo se desarrolló con la finalidad de presentar la demostración del teorema central, de manera detallada, ya que en la literatura de teoría de categorías solamente suele ser mencionada la idea de la prueba. También, es importante mencionar que este trabajo ha sido escrito de tal manera que puede ser entendido por lectores que no estén familiarizados con esta área de la matemática.

5. Agradecimientos. Se quiere expresar un especial agradecimiento al profesor Tomás Núñez Lay por la revisión de la redacción, observaciones y sugerencias de este trabajo; como también al profesor Carlos Sáez Calvo por estar siempre dispuesto a colaborar con algunas interrogantes relacionadas a este trabajo.

ORCID and License

Carlos Mejía Alemán https://orcid.org/0000-0002-5081-9175 Irene Edith Núñez Rodriguez https://orcid.org/0000-0001-7132-0501 
Neisser Pino Romero https://orcid.org/0000-0002-9865-5974

This work is licensed under the Creative Commons - Attribution 4.0 International (CC BY 4.0)

\section{Referencias}

[1] Awodey S. Category Theory, Oxford, Claredndon Press; 2006.

[2] Dubuc EJ. Historia de Categorías. La Gaceta de la RSME. 2014; 17(2):333-347.

[3] Leinster T. Basic Category Theory. Cambridge Studies in Advanced Mathematics, Vol. 143, Cambridge University Press, ISBN 978-1-107-04424-1, 2014. Recuperado de https://arxiv.org/pdf/1612.09375.pdf

[4] MacLane S. Categories for the working mathematician. Second Ed. Springer - Verlag, 1998.

[5] Mejía Alemán C. El lema de Yoneda y algunas de sus aplicaciones.[Tesis de licenciatura]. Lima: Repositorio de tesis digitales, Universidad Nacional Mayor de San Marcos; 2019.

[6] Tondeur P. Categorías y funtores. Cursos y seminarios de matemática, serie A. 2011; Fasciculo 21.

[7] Yokura S. A Remark on Yoneda's Lemma. Math.CTI. 2017. [accesado 20/12/20]; disponible en https://arxiv.org/ abs/1712.02064

[8] Adámek J, Herrlich H, Strecker GE. Abstract and Concrete Categories The Joy of Cats.[En linea]. Bremen, KatMAT Seminar; 2004. [accesado dic. 2021] Disponible en http://katmat.math.uni-bremen.de/acc/acc.pdf 